\title{
Nanosensor and Breath Analyzer for Ammonia Detection in Exhaled Human Breath
}

\author{
Perena Gouma, Krithika Kalyanasundaram, Xiao Yun, Student Member, IEEE, Milutin Stanaćević, Member, IEEE, \\ and Lisheng Wang
}

\begin{abstract}
The detection and monitoring of gases in exhaled human breath up to date has been limited by the lack of appropriate materials and technologies which could rapidly and selectively identify the presence and monitor the concentration of trace levels of specific analytes-biomarkers. We present a metal oxide-based nanosensor that is highly specific to ammonia gas in breath-simulating environments at low part-per-billion concentrations. The design of a handheld breath analyzer for gas detection in exhaled human breath is described. Semiconducting ceramics are presented as suitable sensor materials for easy and affordable non-invasive diagnostics.
\end{abstract}

Index Terms-Chemical sensing, Nanosensors, Breath analyzer, Metal oxide-based sensors, Ammonia detection

\section{INTRODUCTION}

A LTHOUGH analysis of body fluids (blood, sputum, urine) for disease diagnosis and monitoring is routine clinical practice, human breath analysis methodologies that exploit the non-invasive nature of such approach are still under-developed. Since the time of the ancient physician Hippocrates, exhaled breath was recognized as a non-invasive tool to diagnose diseases. Breath testing devices first appeared in 1784 when Lavoisier detected $\mathrm{CO}$ in exhaled breath of guinea pigs [1]. Since then, colorimetric assays and gas chromatography columns have been used to analyze VOCs (volatile organic compounds) in human breath in quantities varying from millimolar $\left(10^{-3} \mathrm{M}\right)$ to picomolar $\left(10^{-12} \mathrm{M}\right)$ concentrations [1]. The latter gas sensitivity limit was achieved by Linus Pauling's gas-chromatography-based breath analysis device in 1971 [2]. Among the 400 compounds of which the human breath consists, only 30 have been identified and most of them are potential indicators (markers) of more than one type of diseases [3]-[5]: $N O$ which has been widely studied as a bio-marker for oxidative stress [6]; exhaled $C O$ also a marker for cardiovascular diseases, diabetes, nephritis, bilirubin production; exhaled hydrocarbons of low molecular

Manuscript received November 15, 2008; revised January 20, 2009. This work is supported by NSF NIRT award and the UHMC Nanomedicine Initiative of SUNYSB on the "Development of a novel breath analysis system".

P. Gouma is with the Department of Materials Science and Engineering, Stony Brook University, Stony Brook, NY 11794-2275 (email:pgouma@notes.cc.sunysb.edu).

K. Kalyanasundaram is with Sensitron, Division of RSM Electron Power, Inc., Deer Park, NY 11729-4681.

X. Yun and M. Stanaćević are with the Department of Electrical and Computer Engineering, Stony Brook University, Stony Brook, NY 117942350.

L. Wang is with the Department of Electrical and Computer Engineering, The University of British Columbia, Vancouver, BC Canada V6T 1 Z4. mass, such as ethane, n-pentane; ethylene, isoprene (hydrocarbon affected by diet which is a marker for blood cholesterol levels) [7]; acetone; formaldehyde; ethanol; hydrogen sulfide, carbonyl sulfides, and ammonia/amines. For example, measurements of exhaled ammonia may differentiate between viral and bacterial infections in lung diseases to justify the use of antibiotics [8], or it may be used to indirectly measure urea levels if monitoring renal diseases [9].

Identifying these signaling metabolites (disease markers) and measuring them in trace concentrations is not a trivial problem. The low concentrations (ppb) of analyte molecules present a major challenge, along with the specificity to a given analyte. On the other hand, the benefit of developing this technology is tremendous. This paper focuses on the synthesis of a nanosensor that can selectively measure ppb concentrations of ammonia gas in breath-simulating environments. In the present work, a breath testing prototype device has also been developed based on an $\mathrm{NH}_{3}$-selective $\mathrm{MoO}_{3}$ nanosensor and it is described in detail. This breath analysis tool operates on the principle of resistive chemosensing, thus it is unique, novel, inexpensive and it rapidly detects ammonia. The selectivity of the nanosensor enables use of simple circuitry for detection and enables design of portable 'cut-off' prototype for prescreening patients with specific diseases. Due to the selectivity of the sensor, pattern recognition algorithms employed with general sensor arrays become obsolete and the conductance of the sensor is directly proportional to specific analyte concentration. Thus to sense the concentration of analyte, the resistance of the sensor is converted to a voltage signal. For a binary response, the percentage increase is compared to a predefined threshold value. The threshold value is set through calibration measurements and the specific LED indicates if the concentration of the analyte has exceeded the threshold value. The prototype device that we have developed is hand-held, offering portability, and it is easy to use.

The paper is organized as follows. Section II reviews the experimental techniques that have been used in the synthesis, characterization, and design of the sensor and the sensing device. Section III describes the experimental results obtained using the nanosensor as well as the operation of the breath analyzer. Concluding arguments are given in Section IV.

\section{EXPERIMENTAL PROCEDURE}

A sol-gel synthesis method [8] was employed to produce 3D networks of $\mathrm{MoO}_{3}$ nanoparticles through an alkoxide reaction between molybdenum isopropoxide and 1-butanol. 


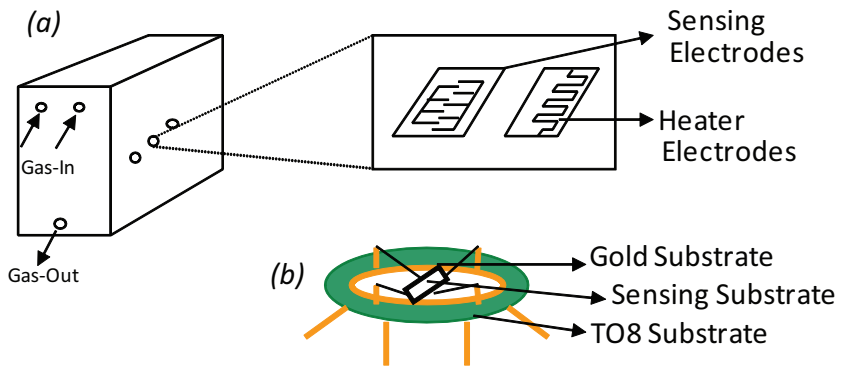

Fig. 1. (a) Schematic of EOS 835 and the sensing chamber; (b) Schematic of the sensing substrate on the TO8 substrate

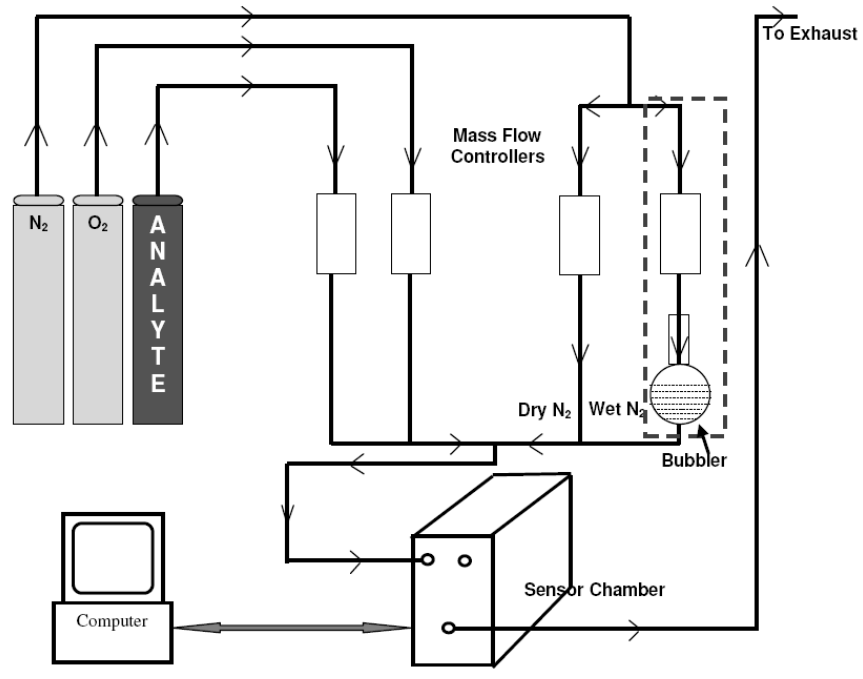

Fig. 2. Schematic of the gas sensing setup

The prepared sol was spin-coated onto sensing substrates ( $3 \mathrm{~mm} \times 3 \mathrm{~mm}$ alumina plated with interdigitated $\mathrm{Pt}$ electrodes produced by our group at Cornell's Nanofabrication Facility) producing thin films of $\mathrm{MoO}_{3}$. The amorphous films were then calcined at $500^{\circ} \mathrm{C}$ for $8 \mathrm{hr}$ to form the $\alpha-M o O_{3}$ polymorph.

The sensor was tested using a gas-flow bench [10]. The gases used were UHP (ultra high purity) nitrogen (Praxair), UHP oxygen (Praxair), $10 \mathrm{ppm}$ ammonia in $N_{2}$ (NORLAB gases). $\mathrm{NH}_{3}$ concentration was varied by varying its flow rates in connection with $\mathrm{N}_{2}$ and $\mathrm{O}_{2}$ flow rates. The gases were controlled through 1479 MKS Mass flow controllers whose channels are connected to a Type 247-MKS 4-channel readout which reads the flow rate of the gases directly in sccm (standard cubic centimeter per minute). The combined flow rate of the gases was maintained at $1000 \mathrm{sccm}$. A schematic of the sensor on a TO8 substrate, the sensing chamber and the gas sensing set up are given in Figure 1 and Figure 2.

The effect of humidity was evaluated using a controlled humidity chamber, in which relative humidity can vary between 0 and $100 \%$ in the presence of the gas under study. Humidity is produced by bubbling $N_{2}$ gas stream through a standard water bubbler at room temperature. The percentage of humidity was controlled by varying the ratio of dry to wet $N_{2}$. A commercially available $\mathrm{CO}_{2}$ filter $(\mathrm{NaOH}$ premixed with
Vermiculite in a 10:1 ratio - Decarbite absorption tube, PW Perkins and Co) was used in these studies. Decarbite reacts only with highly acidic gases such as $\mathrm{CO}_{2}, \mathrm{H}_{2} \mathrm{~S}$.

Furthermore, a portable breath analyzer was developed by attaching a heater to the backside of the sensor's substrate and then embedding them into a circuit board. A breath collection tube is attached to the prototype.

\section{RESUlts AND Discussion}

The sensing element used in this work to detect ammonia was a nanocrystalline, sol-gel processed thin film of a semiconducting ceramic, $\mathrm{MoO}_{3}$. This metal oxide, which is also a known catalyst, has been stabilized in it's $\alpha$-phase polymorph with an orthorhombic crystal structure. The $\alpha-M o O_{3}$ phase has a layered structure with (010) basal plane that is built up of double chains of edge-sharing $\left[\mathrm{MoO}_{6}\right]$ octahedra connected through vertices. Upon reduction in catalytic reaction with gases this phase forms the $\mathrm{Mo}_{18} \mathrm{O}_{52}$ structure instead of the $\mathrm{ReO}_{3}$ type $\mathrm{Mo}_{8} \mathrm{O}_{23}$ shear structure [11]. It was discussed in our earlier publications [10], [12] that the $\alpha$-phase is selective to ammonia and highly sensitive to amines (which are moderate bases) and the sensing mechanism is consistent with the reduction of $\mathrm{MoO}_{3}$ and the formation of ordered phases, which suggests a reaction-based sensing process. This was true for sputtered films [10], as well as for sol-gel processed ones [12]. In all of our previous studies on $\mathrm{MoO}_{3}$ sensors, no matter what the processing method used [13], [14], the $\alpha$-phase exhibits a trend of selective response to ammonia in the presence of interfering gases. Furthermore, this observed selectivity included gases that are typically encountered in the human breath, including $\mathrm{NO}_{2}, \mathrm{NO}, \mathrm{C}_{3} \mathrm{H}_{6}$, and $\mathrm{H}_{2}$; there was a slight cross-sensitivity reported for sol-gel processed films to $C O$ (for tested concentrations between 50-500ppm) [figure 6 in [12]].

In this work, the focus is to demonstrate that the $\alpha-M o O_{3}$ -based sensor may detect ammonia at concentrations relevant to the normal levels found in exhaled human breath. Selected ion flow tube (SIFT) measurements of ammonia in the breath carried out [15] on a sample of normal controls versus a population of stable patients treated for end-stage renal failure show that the former group exhibit ammonia levels of $425 \mathrm{ppb}-$ $1800 \mathrm{ppb}$, mean of $960 \mathrm{ppb}$. The diseased population had ammonia levels on breath ranging from 820 ppb-14700 ppb, with a mean of $4880 \mathrm{ppb}$. The lowest gas concentration we were able to test in our laboratory was $50 \mathrm{ppb}$, so this was the lowest limit of our measurements. Ambient levels of $\mathrm{CO}_{2}$ are around $300 \mathrm{ppm}$ and a typical level in breath is much higher, at approximately $5-6 \%$. At $500^{\circ} \mathrm{C}, \mathrm{CO}_{2}$ is expected to break down to $\mathrm{CO}$ and $\mathrm{O}_{2}$. Given the known cross-sensitivity of the sol-gel sensor to $C O$, a filter is used to remove $C O_{2}$ altogether.

The sensor is semiconducting at elevated temperatures only and the $\alpha$-phase is stabilized above $500^{\circ} \mathrm{C}$. As shown in Figure 3, when the sensor operated at $500^{\circ} \mathrm{C}$ was exposed to various concentrations of $\mathrm{NH}_{3}$ gas in a background mixture of $\mathrm{N}_{2}$ and $\mathrm{O}_{2}(80 \% / 20 \%)$ simulating ambient air, $\mathrm{NH}_{3}$ was detected easily down to $50 \mathrm{ppb}$. When the sensor was exposed 


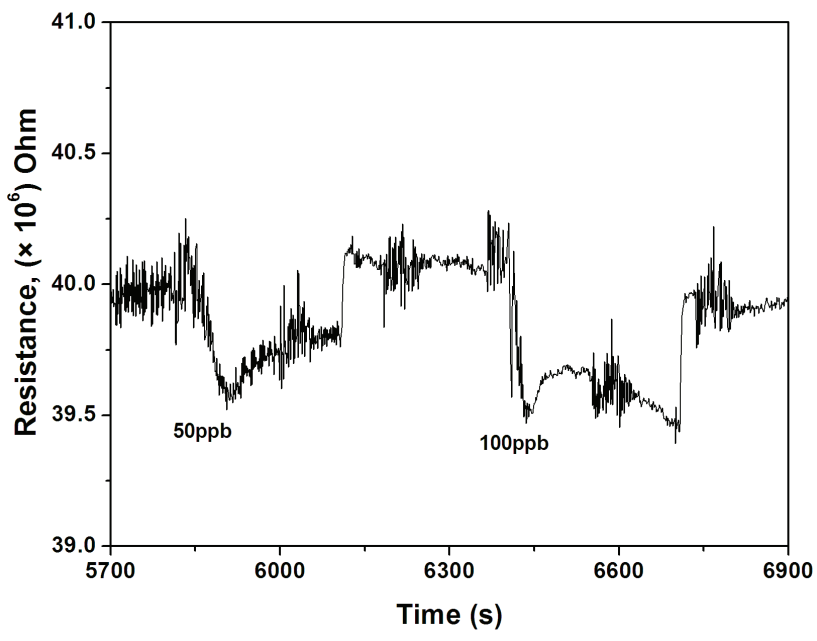

Fig. 3. Sensing response of $\mathrm{MoO}_{3}$ sensor to $\mathrm{NH}_{3}$ (50ppb and 100ppb).

to various concentrations of $\mathrm{NH}_{3}$ and $\mathrm{CO}_{2}$, (using the filter to scavenge $\mathrm{CO}_{2}$ ) in the presence of $\mathrm{N}_{2}$ and $\mathrm{O}_{2}$, the presence of $\mathrm{CO}_{2}$ did not affect $\mathrm{NH}_{3}$ sensing, for concentrations ranging between 0.5 and $10 \mathrm{ppm}$. Such results are clearly shown in Figure $4(a)-(c)$. Preliminary data also showed that up to $25 \%$ humidity in the gas stream does not have any effect on the performance of the sensor (data not shown here). The sensor is reversible; response and recovery times are extremely fast (milliseconds to seconds) and the expected lifetime is over 1 year at the operating temperature.

\section{A. Binary Prototype}

The goal of the designed sensor prototype is to provide measurement and quick 'cut-off' response display. For the quick initial pre-screen procedure, the qualitative measurement of the gas concentration is not necessary. LED display is implemented to indicate the binary measurement results. Thus, when the output resistance is higher than a predefined higher threshold, a red LED is lit up; when the output resistance is lower than a predefined lower threshold, a green LED is lit up; when the output resistance stays in the middle, both LEDs are off.

A schematic of designed prototype is shown in Figure 5. The output of the sensor is connected with a fixed resistor serially. With the help of the voltage divider, the output resistance is converted to a voltage signal, which is proportional to the output signal. The voltage signal, indicated as $V_{\text {test }}$ in the figure, is connected to a comparator and compared to a threshold voltage, $V_{t h}$, which is created by another voltage divider. A potentiometer is used to create the threshold voltage, so that it is very easy to adjust the threshold. The comparator compares the two inputs and generates a output signal that either turns on or turns off the following LED.

For simplicity, Figure 5 only shows the comparison with higher threshold. If $V_{\text {test }}$ is higher than $V_{t h}$, the output of comparator is high, thus turns on the red LED. The same schematic is applied for the comparison with lower threshold, where the only difference is $V_{\text {test }}$ is now connected to the negative terminal of comparator, and $V_{t h}$, which is the lower

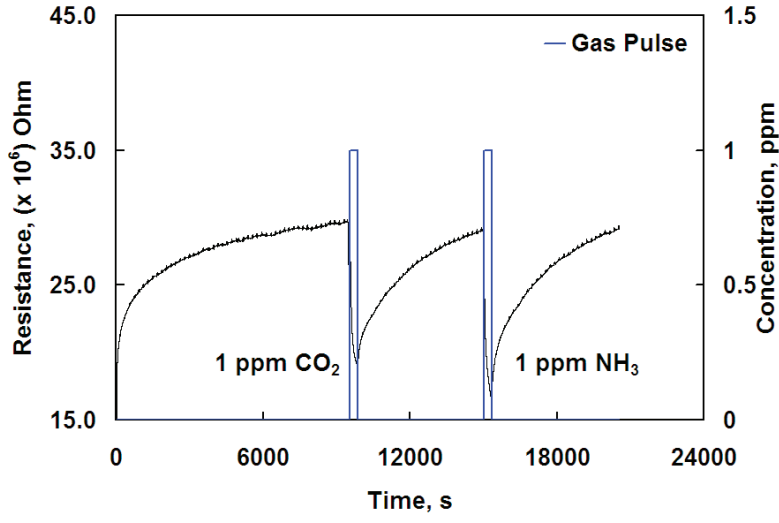

(a)

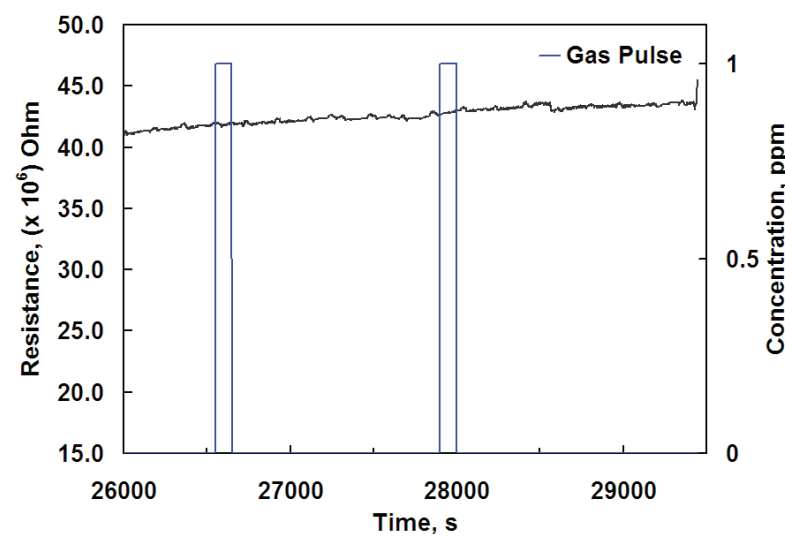

(b)

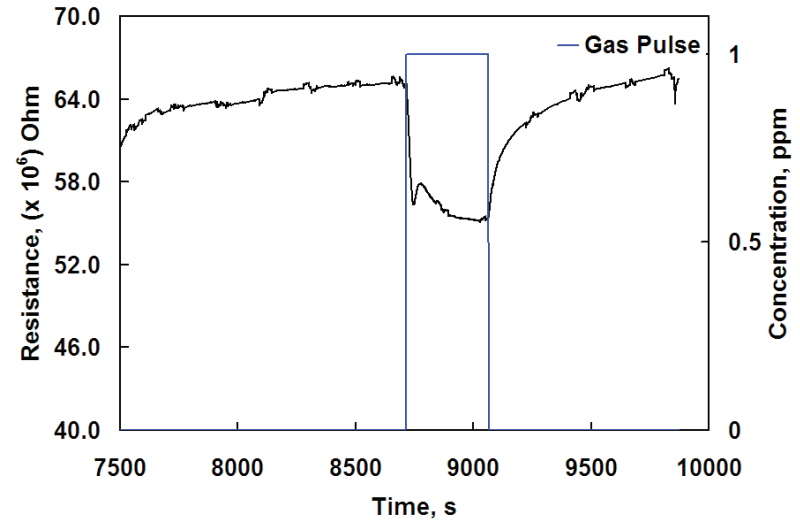

(c)

Fig. 4. Sensing responses of the $\mathrm{MoO}_{3}$ sensor to lppm concentration of (a) $\mathrm{CO}_{2}$ and $\mathrm{NH}_{3}$ without filter; (b) $\mathrm{CO}_{2}$ with filter; and (c) $\mathrm{NH}_{3}$ with filter.

threshold here, is connected to the positive terminal. In addition, connections for the on-chip heater is also provided in the prototype.

Figure 6 shows a photograph of the designed binary sensor prototype. The $\mathrm{MoO}_{3}$ sensor is isolated from the environment by a chamber. A specially designed channel allows exhaled human breath flow or controlled gas flow to go through the chamber and interact with the sensor (not shown here). The mouthpiece receives the analyte input. The sensor determines the concentration of $\mathrm{NH}_{3}$. Based on predetermined threshold, the result is displayed either as a red (positive for high 


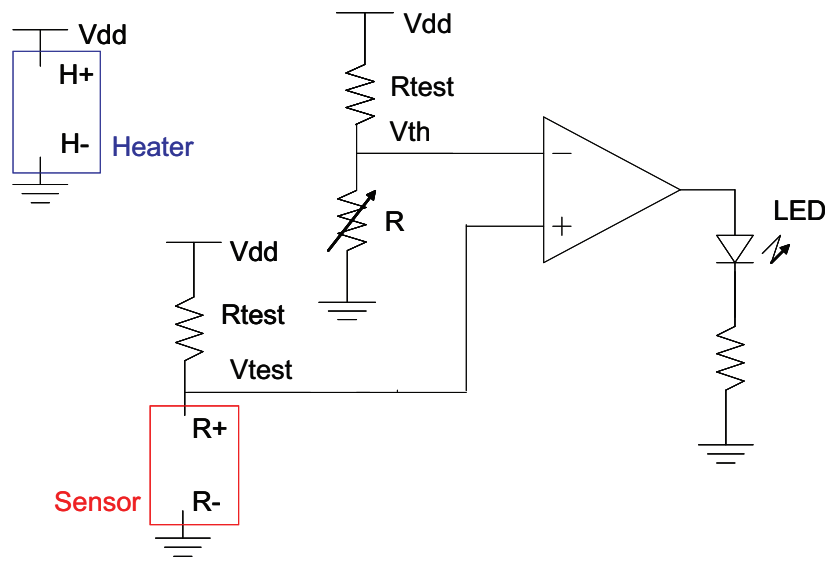

Fig. 5. Schematic diagram of the designed prototype.

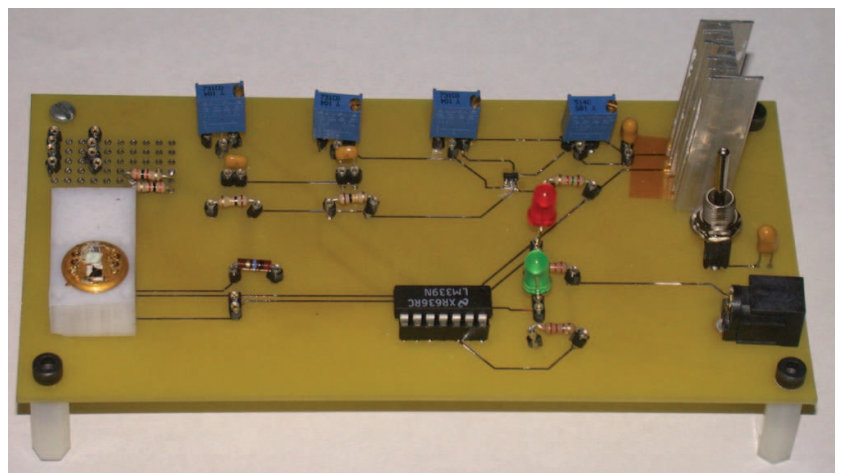

Fig. 6. Photograph of designed binary portable prototype for detection of signaling metabolites.

ammonia level indicating diseased state) or green (negative for disease) light signal. The prototype is powered with an external power adaptor, and regulated with an on-board adjustable voltage regulator. The adjustable voltage regulator is chosen to enable testing of the sensor with different biasing voltage. The comparators are implemented with commercially available ICs, LM339 from National Semiconductor. Due to the high current, up to $450 \mathrm{~mA}$, required from the heater, a heat sink is used to help the regulator work properly.

Our prototype system was developed to validate the feasibility of breath analyzer and selectively to sense the range of concentrations of $\mathrm{NH}_{3}$ that could be present in the human breath. The designed prototype will be used in future medical trials that would determine the appropriate threshold value.

\section{CONCLUSION}

This paper reports on a ceramic nanosensor for the selective detection of ppb levels of ammonia in breath-simulating environments and on the design of a simple breathalyzer prototype device utilizing such chemiresistive sensors for fast, convenient, inexpensive, and non-invasive monitoring of exhaled gases in human breath for disease diagnosis. The ceramic (metal oxide) science and technology reported here may revolutionize nanomedicine applications, such as inexpensive and easy to use non-invasive diagnostic tests.

\section{REFERENCES}

[1] M. Phillips, "Detection of Volatile Organic Compounds in Breath," in Disease Markers in Exhaled Breath, N. Marczin, S.A. Kharitonov, M.H. Yacoub, and P.J. Barnes, Eds., New York: Marcel Decker, 2002. pp. 219-231.

[2] V.E. Arterbery, W.A. Pryor, L. Jiang, S.S. Sehnert, W.M. Foster, R.A. Abrams, J.R. Williams, M.D. Wharam, Jr. and T.H. Risby, "Breath Ethane Generation during Clinical Total Body Irradiation as a Marker of OxygenFree-Radical-Mediated Lipid Peroxidation: A Case Study," Free Radical Biol. Med., vol. 17 (6), pp. 569-576, 1994.

[3] S.A. Kharitonov and P.J. Barnes, "Exhaled Markers of Pulmonary Disease: State of the Art," Am. J. Respir. Cell Mol. Biol., vol. 163 (9), pp. 1693-1722, 2001

[4] A.D. Smith, J.O. Cowan, S. Filsell, C. McLachlan, G. Monti-Sheehan, P. Jackson, and D.R. Taylor, "Diagnosing Asthma: Comparisons between Exhaled Nitric Oxide Measurements and Conventional Tests," Am. J. Respir. Cell Mol. Biol., vol. 169 (4), pp. 473-478, 2004.

[5] S.M. Studer, J.B. Orens, I. Rosas, J.A. Krishman, K.A. Cope, S. Yang, J.V. Conte, P.B. Becker, and T.H. Risby, "Patterns and Significance of Exhaled-Breath Biomarkers in Lung Transplant Recipients with Acute Allograft Rejection," J. Heart Lung Transplant, vol. 20 (11), pp. 11581166, 2001.

[6] T.H. Risby and S.S. Sehnert, "Clinical Application of Breath Biomarkers of Oxidative Stress Status," Free Radical Biol. Med., vol. 27 (11-12), pp. 1182-1192, 1999.

[7] T. Karl, P. Prazeller, D. Mayr, A. Jordan, J. Rieder, R. Fall, and W. Lindinger, "Human Breath Isoprene and Its Relation to Blood Cholesterol Levels: New Measurements and Modeling," J. Appl. Physiol., vol. 91 (2), pp. 762-770, 2001

[8] S.A. Kharitonov and P.J. Barnes, "Biomarkers of Some Pulmonary Diseases in Exhaled Breath," Biomarkers, vol. 7 (1), pp. 1-32, 2002.

[9] K. Sawicka, P. Gouma and S. Simon, "Electrospun Biocomposite Nanofibers for Urea Biosensing," Sens. Actuators, B, vol. 108 (1-2), pp. 585-588, 2005.

[10] A.K. Prasad, P.I. Gouma, D. J. Kubinksi, J.H. Visser, R.E. Soltis, and P.J. Schmitz, "Reactively Sputtered $\mathrm{MoO}_{3}$ Films for Ammonia Sensing," Thin Solid Films, vol. 436 (1), pp. 46-51, 2003.

[11] T. Ressler, J. Wienold J, R.E. Jentoft and T. Nelsius, "Bulk Structural Investigation of the Reduction of $\mathrm{MoO}_{3}$ with Propene and the Oxidation of $\mathrm{MoO}_{2}$ with Oxygen," J. Catal., vol. 210 (1), pp. 67-83, 2002.

[12] A.K. Prasad, D. Kubinski, and P. I. Gouma, "Comparison of Sol-Gel and Ion-Beam Deposited $\mathrm{MoO}_{3}$ Thin Film Gas Sensors for Selective Ammonia Detection," Sens. Actuators, B, vol. 9, pp. 25-30, 2003.

[13] A.K. Prasad, "Study of gas specificity in $\mathrm{MoO}_{3} / \mathrm{WO}_{3}$ thin film sensors and their arrays," Ph.D. Thesis, SUNY Stony Brook, NY, 2005.

[14] K. Kalyanasundaram, "Biomarker Sensing using Nanostructured Metal Oxide Sensors," Ph.D. Thesis, SUNY Stony Brook, NY, 2007.

[15] S. Davies, P. Spanel and D. Smith, "Quantitative Analysis of Ammonia on the breath of patients in end-stage renal failure," Kidney International, vol. 52, pp. 223-228, 1997.

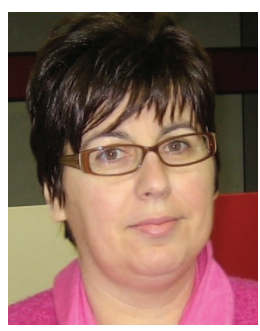

Perena Gouma is an Associate Professor in the Department of Materials Science and Engineering at the State University of New York-Stony Brook and the Director of the Center for Nanomaterials and Sensor Development. She is a Fulbright Fellow and a US delegate for the National Academies' GDEST Workshop on the Future of Sensors and Sensor Systems. She has published over 90 peer-reviewed articles, she has been granted several patents on biochemical sensor technologies. She is an Associate Editor of the Journal of the American Ceramic Society, and serves on the editorial boards of several other journals on sensors and nanomedicine. Her research interests include nanostructured and nanocomposite materials for selective chemosensors, electronic noses, and breathanalyzers for non-invasive diagnostics. 


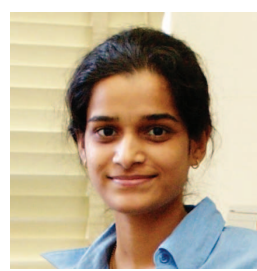

Krithika Kalyanasundaram received her Bachelors in Engineering in Metallurgy from National Institute of Technology, Tiruchirapally (formerly Regional Engineering College), India in 2003 and PhD in Materials Science and Engineering from SUNY, Stony Brook, in 2007. She currently works as a Sr. Materials Engineer for Sensitron Semiconductor, Deer Park, NY. Her research interests include nanomaterials, structure property correlations in metal oxide semiconductors, gas sensors, selective gas sensing, breath analysis, and electron microscopy.

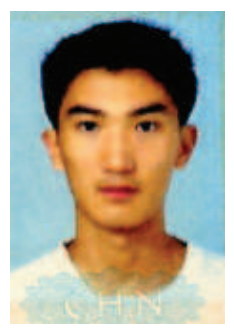

Xiao Yun (S'06) received the B.S. degree in Electrical Engineering from the Beijing University of Aeronautics and Astronautics, Beijing in 2005. He is currently a PhD candidate of Electrical and Computer Engineering at Stony Brook University, NY. His research interests include low-power low-noise mixed-signal VLSI circuits for sensor applications.

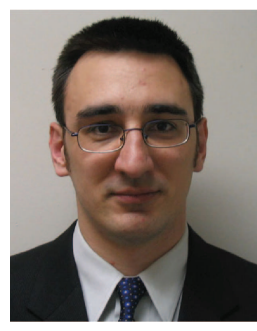

Milutin Stanaćević (S'00-M'05) received the B.S. degree in Electrical Engineering from the University of Belgrade, Serbia in 1999, and the Ph.D. degree in Electrical and Computer Engineering from Johns Hopkins University, Baltimore, MD, in 2005. He is an Assistant Professor of Electrical and Computer Engineering at Stony Brook University, NY. His research interests include mixed-signal VLSI circuits, systems, and algorithms for parallel multi-channel sensory information processing with emphasis on real-time acoustic source localization and separation, and micropower implantable biomedical instrumentation and telemetry.

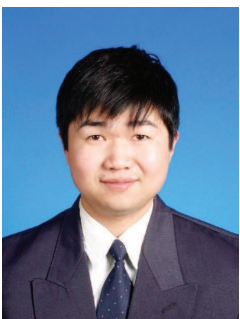

Lisheng Wang received his B. Eng. degree in Materials Science and Engineering from Tsinghua University, China in 2003 and the M. Eng. from the same institution in 2005. He received his Ph.D. degree in Materials Science and Engineering from Stony Brook University, NY USA in 2008. He is currently a postdoctoral fellow in University of British Columbia, Canada. His research interests include nanostructured metal oxides, polymers and their composites, design of gas/VOC sensitive devices based on these materials for environmental monitoring, human disease diagnosis and energy resource detection. 